\title{
Relationship between displacement and velocity amplitudes of seismic waves from local earthquakes
}

\author{
Akio Katsumata \\ Meteorological College, JMA, 7-4-81 Asahi-cho, Kashiwa, Chiba 277-0852, Japan \\ (Received February 2, 2000; Revised March 2, 2001; Accepted March 2, 2001)
}

\begin{abstract}
Relationship between displacement and velocity amplitudes of seismic waves was examined with data at stations within $200 \mathrm{~km}$ from 142 local earthquakes in and near Japan. The expected value of the coefficient for the logarithmic velocity amplitude to the logarithmic displacement amplitude is 0.5 when a self-similar scaling model is assumed. Observed value of the coefficient is about $0.8 \sim 0.9$. This value appears to be valid at least in the magnitude range from 3.0 to 6.5. Although a spectral model simulation suggested that apparent large contents of high-frequency components were required to explain the observed coefficient, no distinct deviation from the $\omega$-square model was found in the observed spectral ratios from earthquakes of different sizes, for which the path effects were virtually excluded. By using an empirical Green's function which would correct the effects of propagation and site amplification, it was shown that the apparent deviation from the self-similar scaling model was due to propagation effects.
\end{abstract}

\section{Introduction}

Magnitude was originally defined as the logarithm of the maximum displacement amplitude (Richter, 1935; Gutenberg, 1945a, b, c). There have been many investigations on the relationships between magnitudes and various seismic parameters. Seismic moment is one of the most important source parameters. Kanamori (1977) introduced moment magnitude, $M_{w}$, for which magnitude and the logarithmic scalar moment is related with a coefficient of 1.5 .

The author focus on the relationships among logarithmic displacement and velocity amplitudes, and magnitude in this paper. This is related to an attempt of determining a magnitude from regional velocity amplitudes.

Relationship between magnitude and ground velocity has been examined to estimate peak ground velocity $(P G V)$ from earthquake magnitude for disastrous earthquakes. Several formulas have been proposed to express the relationship between velocity amplitude and earthquake magnitude. Joyner and Boore (1981) examined the relationship between $M_{w}$ and peak horizontal acceleration and velocity. They obtained a value of 0.489 to relate $M_{w}$ to the logarithmic $P G V$. Campbell (1997) presented relationships between $M_{w}$ and peak ground acceleration and velocity, in which a value of 0.51 is used to relate $M_{w}$ to the logarithmic $P G V$. Using stiff-site peak velocity $P G V_{S}$, Midorikawa (1993) adopted a quadratic formula to express the relationship between $P G V_{S}$ and $M_{w}$.

Molas and Yamazaki (1995) obtained a formula for the relationship between ground velocity in Japan and magnitude determined by the Japan Meteorological Agency (JMA), in which the estimated coefficient of the JMA magnitude to the

Copy right (C) The Society of Geomagnetism and Earth, Planetary and Space Sciences (SGEPSS); The Seismological Society of Japan; The Volcanological Society of Japan; The Geodetic Society of Japan; The Japanese Society for Planetary Sciences. logarithmic $P G V$ was 0.628 . The JMA magnitude $\left(M_{J M A}\right)$ for shallow earthquakes was determined with Tsuboi's formula (1954). Utsu (1982) reported that average difference between $M_{J M A}$ and $M_{w}$ is less than 0.15 in the magnitude range from 4.5 to 7.5 for shallow earthquakes.

Relationship between $M_{J M A}$ and velocity amplitude has been examined by some authors for determining magnitude from velocity amplitudes obtained from short-period seismometers. Values of 0.85 (Watanabe, 1971), 0.78 (Yoshioka and Iio, 1988), and 1/1.27 (0.79) (Kanbayashi, 1992) were used to express relationship between $M_{J M A}$ and the logarithm of the maximum velocity amplitude. Kakishita et al. (1992) showed data which supported the result by Watanabe (1971).

The relationship between the seismic moment and magnitude calculated from displacement amplitudes has been studied mainly in the frequency domain. Aki (1967) explained the relationship between seismic moment and $M_{S}$, and derived the $\omega$-square model. Boore (1983) explained the relationship between $M_{w}$ and $P G V$ by the stochastic ground motion simulation based on the $\omega$-square model.

In this study, the relationship between displacement and velocity amplitudes is examined using acceleration recorded at stations of a JMA regional network. The meaning of the scaling factor which relates the logarithmic velocity amplitude to the logarithmic displacement amplitude is discussed. This work is intended for giving a theoretical base of a magnitude calculated from regional velocity amplitudes, and for inspecting validity of the $\omega$-square model for small and moderate-sized earthquakes.

\section{Comparison between Displacement Amplitude and Velocity Amplitude}

The acceleration records were obtained with JMA 87-type 
electromagnetic strong motion seismographs, which record ground acceleration up to $9.8 \mathrm{~m} / \mathrm{s}^{2}$ with the resolution down to $3.0 \times 10^{-4} \mathrm{~m} / \mathrm{s}^{2}$, the sampling rate of $50 \mathrm{~Hz}$, and the frequency range of $0.001-10 \mathrm{~Hz}$ (Japan Meteorological Agency, 1989; Kakishita et al., 1992). The analyzed data were obtained at 74 stations on the Japanese islands (Kakishita et al., 1992) for 142 earthquakes from August, 1988 to July, 1993. The magnitude range was 4.0 to 7.0. The trapezoid formula was used for the integration to obtain displacement and velocity amplitudes from acceleration records. A 3rd-degree Bessel high-pass filter (Katsumata, 1993) of $0.1 \mathrm{~Hz}$ cut-off was applied after the integration. Acceleration of $3.0 \times 10^{-4}$ $\mathrm{m} / \mathrm{s}^{2}$ at $0.1 \mathrm{~Hz}$ corresponds to the velocity of $4.8 \times 10^{-4} \mathrm{~m} / \mathrm{s}$ and the displacement of $7.8 \times 10^{-4} \mathrm{~m}$. Noisy parts due to the digitizing noise were rejected in measuring amplitudes. The hypocentral distance was restricted to $200 \mathrm{~km}$. The data distribution of epicentral distance and focal depth is shown in Fig. 1.

Suppose that relationships among $M_{w}$, the maximum displacement amplitude, $A_{D}(\mathrm{~m})$, the maximum velocity amplitude, $A_{V}(\mathrm{~m} / \mathrm{s})$, and epicentral distance or hypocentral distance, $R(\mathrm{~km})$, can be approximately expressed as

$$
\begin{aligned}
& \log _{10} A_{D}=\alpha_{D} M_{w}+\beta_{D} \log _{10} R+\gamma_{D} \\
& \log _{10} A_{V}=\alpha_{V} M_{w}+\beta_{V} \log _{10} R+\gamma_{V},
\end{aligned}
$$

where $\alpha_{D}, \beta_{D}, \gamma_{D}, \alpha_{V}, \beta_{V}$, and $\gamma_{V}$ are constants. When $A_{D}$ is half the maximum peak-to-peak amplitude of any phases at stations within $2000 \mathrm{~km}$ from epicenters, Eq. (1) with the values of $\alpha_{D}=1.0, \beta_{D}=-1.73$, and $\gamma_{D}=-5.17$ (Tsuboi, 1954) gives a good approximation for magnitude from 5 to 7 (Katsumata, 1996).

By using the above formulas, the relationship between displacement and velocity amplitudes is

$$
\begin{aligned}
\log _{10} A_{V}= & \frac{\alpha_{V}}{\alpha_{D}} \log _{10} A_{D}+\left(-\frac{\alpha_{V}}{\alpha_{D}} \beta_{D}+\beta_{V}\right) \log _{10} R \\
& +\left(-\frac{\alpha_{V}}{\alpha_{D}} \gamma_{D}+\gamma_{V}\right) \\
= & \alpha^{\prime} \log _{10} A_{D}+\beta^{\prime} \log _{10} R+\gamma^{\prime} .
\end{aligned}
$$

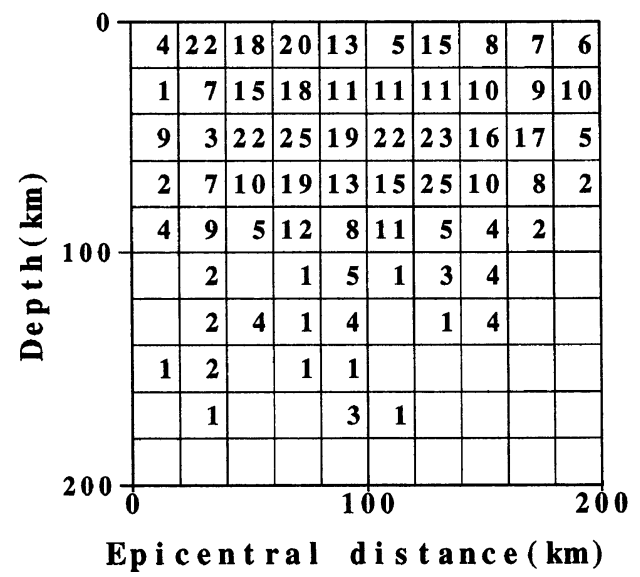

Fig. 1. Data distribution in epicentral distance and focal depth. Numbers denote the size of data points in each $25 \mathrm{~km} \times 25 \mathrm{~km}$ grid of epicentral distance and focal depth. Data within $200 \mathrm{~km}$ from hypocenters is used in the analysis. A total of 592 amplitudes from 142 earthquakes are used.
The resultant of half the peak-to-peak amplitude of the two horizontal components, $A=\sqrt{A_{N S}^{2}+A_{E W}^{2}}$, is used here for the maximum amplitude, where $A_{N S}$ and $A_{E W}$ are half the maximum peak-to-peak amplitudes from the traces of the horizontal components. This type of compound was used by Tsuboi (1954).

The values of $\alpha^{\prime}, \beta^{\prime}$, and $\gamma^{\prime}$ were estimated to examine the relationship between displacement and velocity amplitudes. An empirical relationship between $\log _{10} A_{D}$ and $\log _{10} A_{V}-$ $\beta^{\prime} \log _{10} R\left(=\log _{10}\left(A_{V} / R^{\beta^{\prime}}\right)\right)$ is shown in Fig. 2. The linear relationship suggests that the least squares method can be used to obtain unknown parameters in Eq. (3). The least squares estimation gave values of $\alpha^{\prime}, \beta^{\prime}$, and $\gamma^{\prime}$ as

$$
\begin{aligned}
\alpha^{\prime} & =0.90 \\
\beta^{\prime} & =-0.29 \\
\gamma^{\prime} & =1.22 .
\end{aligned}
$$

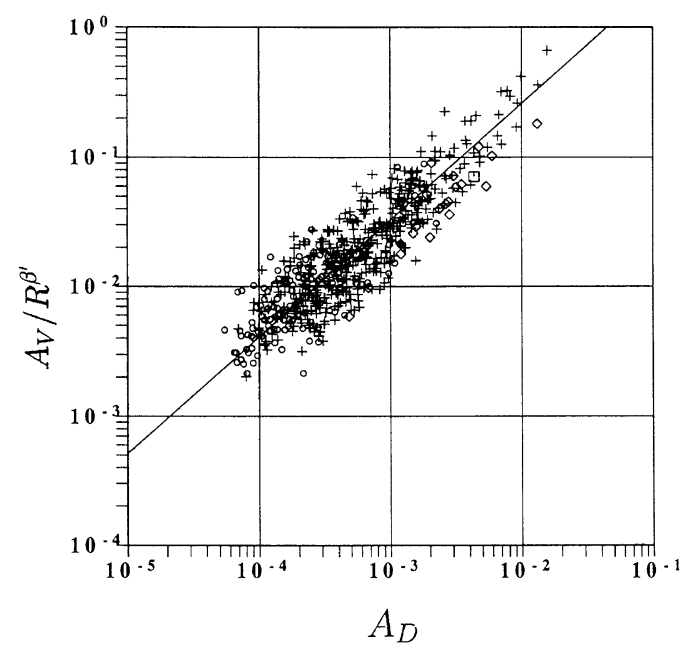

Fig. 2. Relationship between maximum displacement amplitude, $A_{D}$, and maximum velocity amplitude converted for hypocentral distance, $A_{V} / R^{\beta^{\prime}}$. Parameter $\beta^{\prime}$ comes from Eq. (3). Symbol kinds denote magnitude ranges: square, $M \geq 7.0$; diamond, $6 \leq M<7$; cross, $5 \leq M<6$; circle, $M<5$.

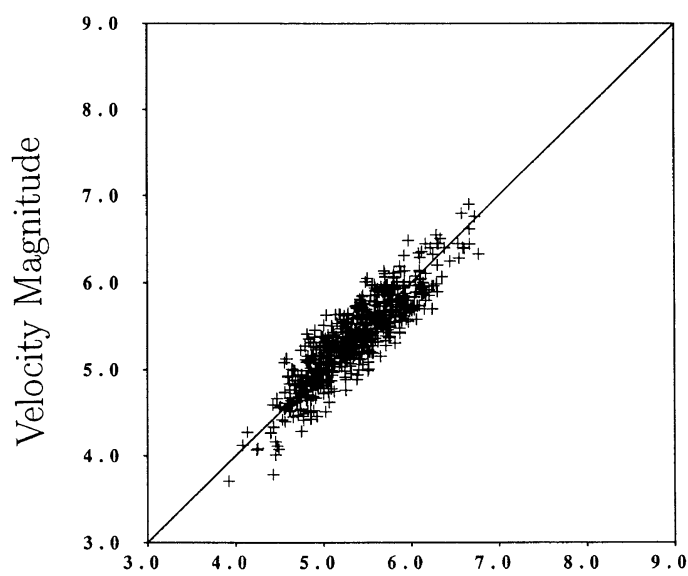

Displacement Magnitude

Fig. 3. Comparison of magnitudes calculated from displacement and velocity amplitudes. 
(a) Windowed white noise

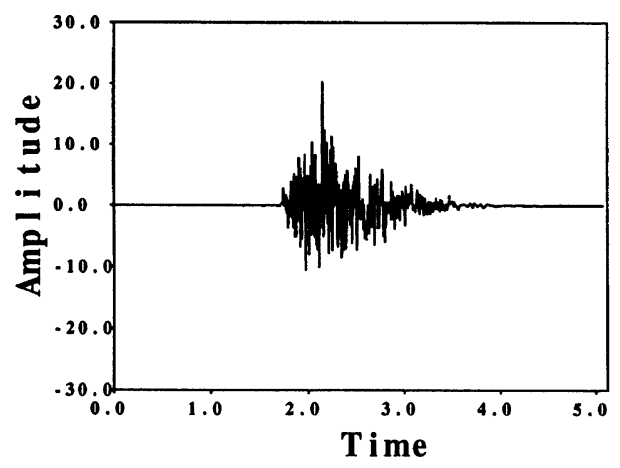

(c) Source time function

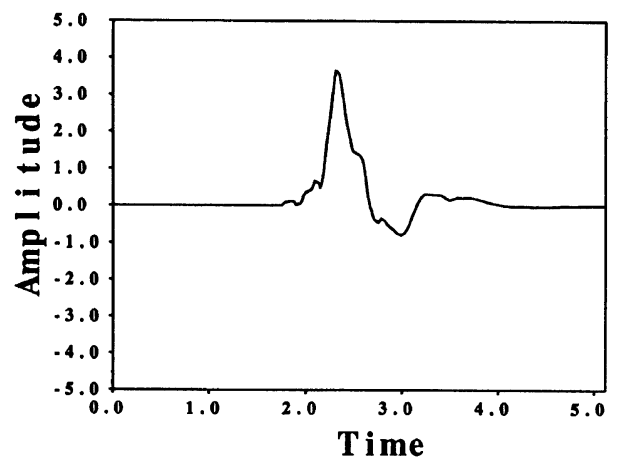

(b) Source spectrum

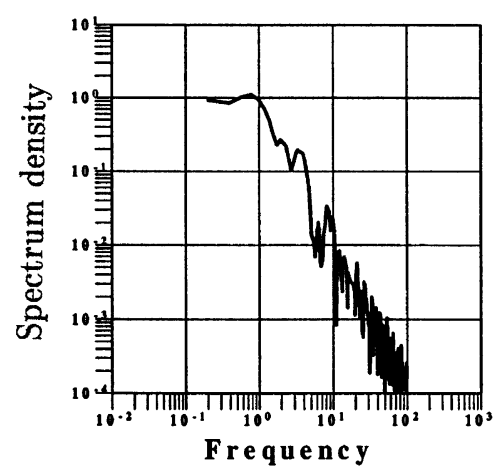

(d) Time derivative of (c)

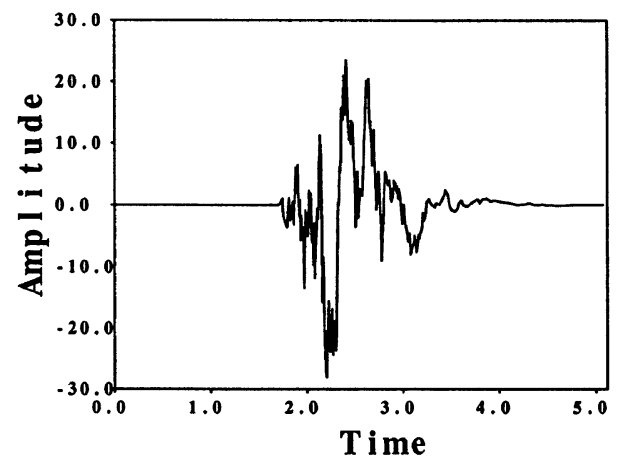

Fig. 4. Synthesizing a transient time series which has specified spectrum density. The method proposed by Boore (1983) is used here. (a) A trace of windowed white noise. The trace of white noise is obtained by inverse-Fourier-transforming a spectrum with unit amplitude and random phase for all frequencies. (b) Spectrum amplitude deformed by a specific transfer function. (c) A displacement trace obtained from the spectrum of (b) by inverse Fourier transform. (d) A velocity trace obtained by differentiating the trace of (c).

(a)

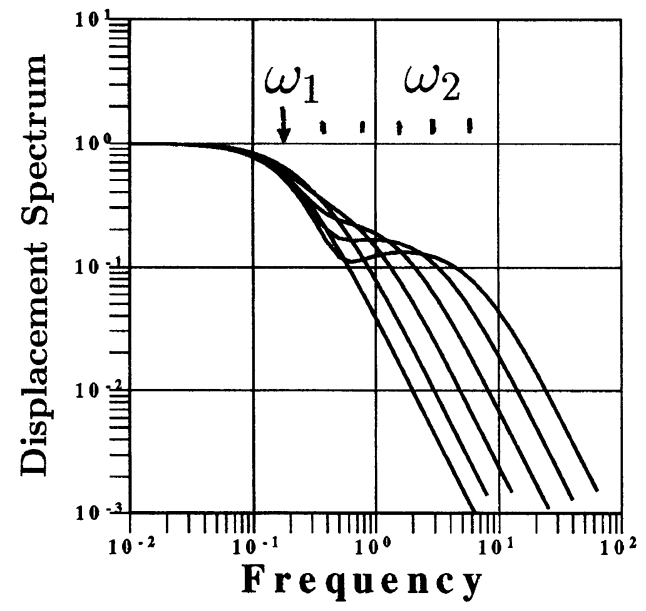

(b)

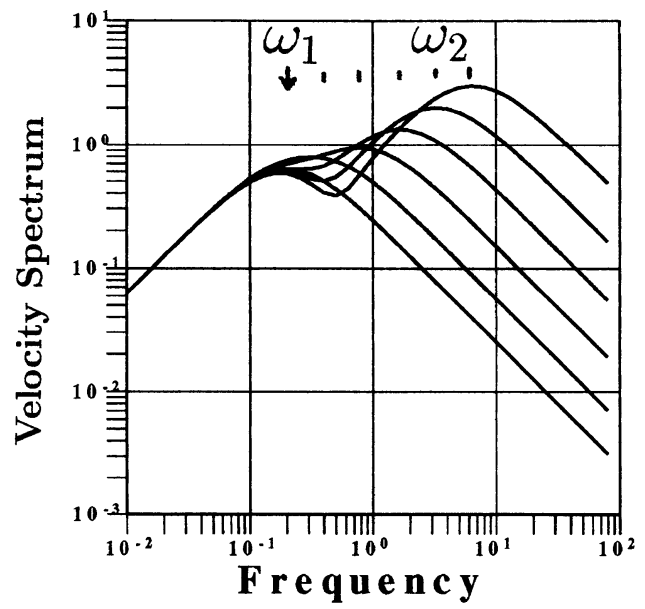

Fig. 5. The spectrum model expressed by Eq. (7): (a) displacement spectrum; (b) velocity spectrum. The value of $s$ in Eq. (8) is assumed 0.5 . The axes show relative values of frequency and spectrum.

The root mean squared (RMS) error of the data is 0.21 . The covariance matrix obtained with the linear least squares method provides a measure of the estimation error.

$$
\begin{aligned}
& \left(\begin{array}{ccc}
\sigma_{\alpha^{\prime} \alpha^{\prime}} & \sigma_{\alpha^{\prime} \beta^{\prime}} & \sigma_{\alpha^{\prime} \gamma^{\prime}} \\
& \sigma_{\beta^{\prime} \beta^{\prime}} & \sigma_{\beta^{\prime} \gamma^{\prime}} \\
& & \sigma_{\gamma^{\prime} \gamma^{\prime}}
\end{array}\right) \\
& =\left(\begin{array}{ccc}
0.00034 & 0.00025 & 0.00062 \\
& 0.00182 & -0.00286 \\
& & 0.00792
\end{array}\right) \text {. }
\end{aligned}
$$




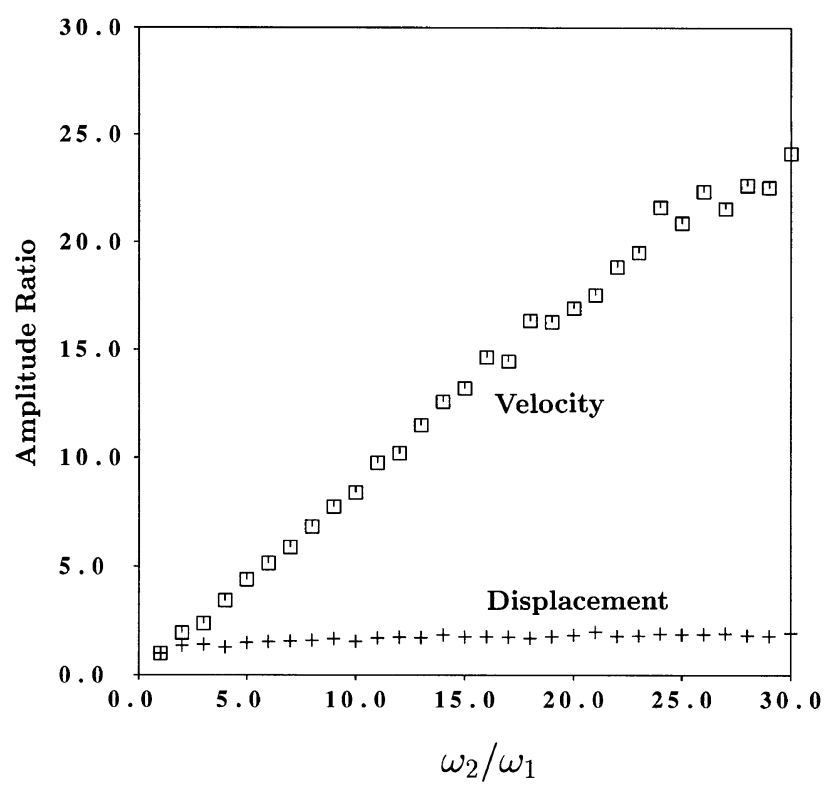

Fig. 6. Relationship between $\omega_{2} / \omega_{1}$ and the peak amplitudes calculated using Eq. (7) with the value $s=0.5$. The horizontal axis shows the ratio of $\omega_{2} / \omega_{1}$, and the vertical axis the ratio of each amplitude to that of $\omega_{2} / \omega_{1}=1.0$. A cross shows the ratio of peak displacement, and a square the ratio of peak velocity.

The estimation error of $\alpha^{\prime}$ is $\sqrt{\sigma_{\alpha^{\prime} \alpha^{\prime}}}=0.018$.

If half the maximum peak-to-peak amplitude of the vertical component is used for $A_{V}$, the parameters become

$$
\begin{aligned}
\alpha^{\prime} & =0.83 \\
\beta^{\prime} & =-0.28 \\
\gamma^{\prime} & =0.37 .
\end{aligned}
$$

The RMS error of data and $\sqrt{\sigma_{\alpha^{\prime} \alpha^{\prime}}}$ are 0.19 and 0.018 , respectively. The value $\alpha_{V}=0.83$ is close to 0.85 obtained by Watanabe (1971) for Tsuboi's magnitude (1954).

In Fig. 3, a comparison of station magnitudes calculated from displacement and velocity amplitudes is shown. The magnitudes are based on Tsuboi's formula and a formula as

$$
M=\left(\log _{10} A_{V}-\beta_{V} \log _{10} R-\gamma_{V}\right) / \alpha_{V},
$$

where the amplitude of the vertical component is used as $A_{V}$, and the values in Eq. (4) are applied in this analysis. A linear relationship is seen between $\left(\log _{10} A_{D}-\beta_{D} \log _{10} R-\right.$ $\left.\gamma_{D}\right) / \alpha_{D}$ and $\left(\log _{10} A_{V}-\beta_{D} \log _{10} R-\gamma_{D}\right) / \alpha_{V}$ in the magnitude range from 4.5 to 6.5 . The value of Watanabe (1971) is estimated by using data primarily within magnitude range from 3 to 5 . The value of $\alpha_{V} \simeq 0.83$ is considered valid for the magnitudes from 3 to 6.5 .

\section{Scaling Models}

The expected difference between coefficients for logarithmic displacement and velocity amplitudes is discussed in this section based on simple scaling models. In this discussion, we assume that the observed values result only from the seismic sources, and that they do not reflect site effects.

At the first, a self-similar model is examined. If the averaged stress drop and the aspect ratio of the fault are as-

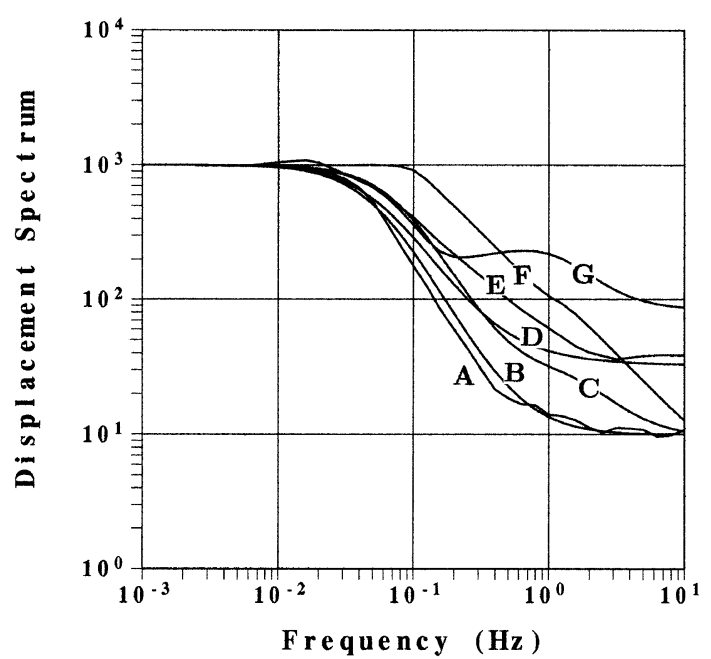

Fig. 7. Comparison of spectrum models. The curves shows the ratio between the source spectrum density for $M_{w}=6.6$ and that for $M_{w}=4.6$. A, Koyama (1985) with parameters of $\zeta=6.7$ and $\epsilon=1.0$; B, Aki (1967); C, Atkinson and Boore (1995); D, model B of Aki (1972); E, Gusev (1983); F, Haddon (1996); G, this model. The size of $M_{w}=4.6$ is the minimum shown by Gusev (1983).

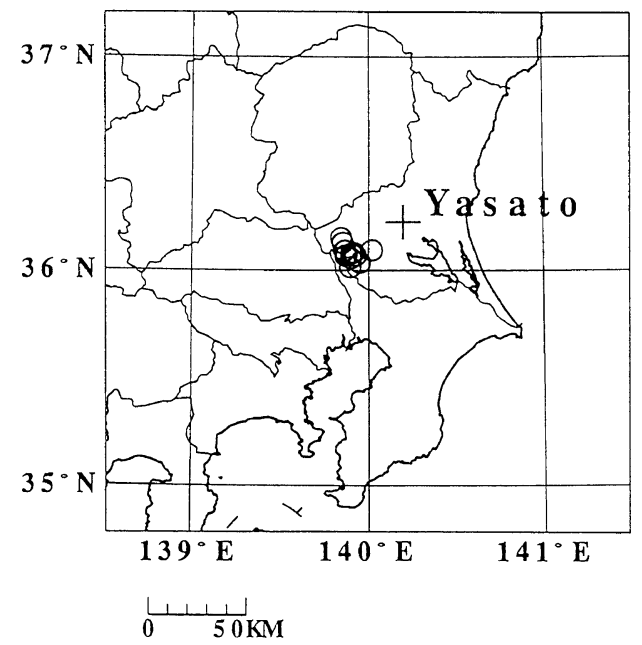

Fig. 8. Locations of Yasato station, where analyzed seismic records were obtained, and of the epicenters of analyzed earthquakes.

sumed to be common for any size of earthquakes, the duration of faulting process of an earthquake $T$ is proportional to $M_{0}^{1 / 3}$ (e.g., Brune, 1970). Body-wave amplitude is proportional to the time derivative of released moment (e.g., Aki and Richards, 1980). It is assumed here that a source time function of far-filed displacement, $f\left(t, M_{0}\right)$, is expressed as

$$
f\left(t, M_{0}\right)=M_{0}^{2 / 3} g\left(t / M_{0}^{1 / 3}\right),
$$

where $g(t)$ is a function of time $t$, and $M_{0}$ is seismic moment. When the spectrum of $g(t)$ shows $\omega^{-2}$-type decay at high frequencies, $f\left(t, M_{0}\right)$ should be consistent with the $\omega$-square model. The integral of $f\left(t, M_{0}\right)$ is proportional to $M_{0}$.

$$
\int_{0}^{\infty} f\left(t, M_{0}\right) d t=M_{0} G_{0},
$$




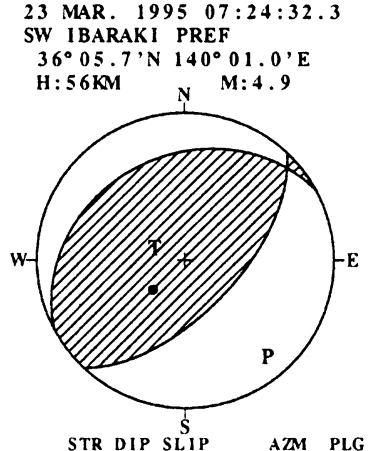

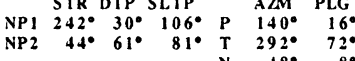

1 JUL. $1997 \quad 18: 00: 17.5$ SW IBARAKI PREF

$36^{\circ} 04.3^{\prime} \mathrm{N} 139^{\circ} 52.4^{\prime} \mathrm{E}$

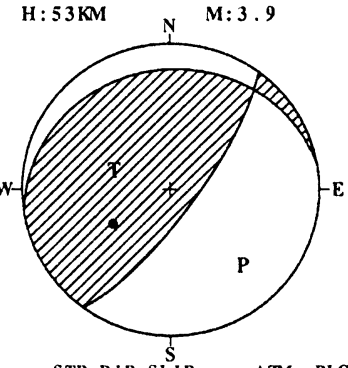

STR DIP SLIP AZM PLG

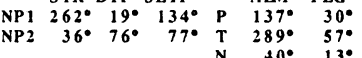

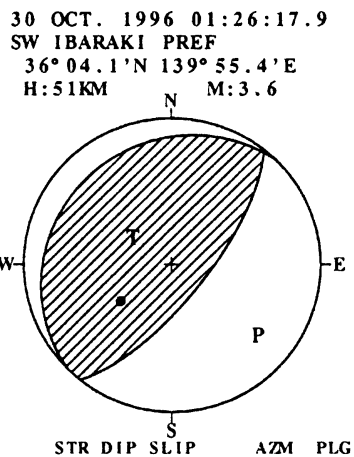

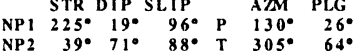

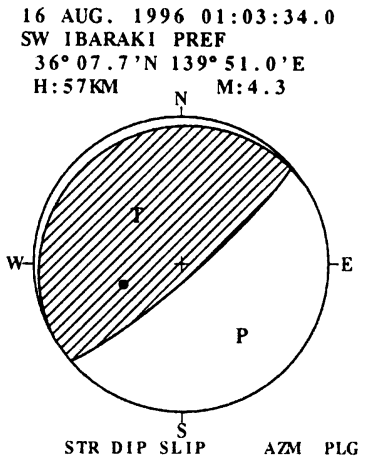

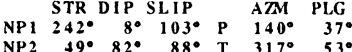
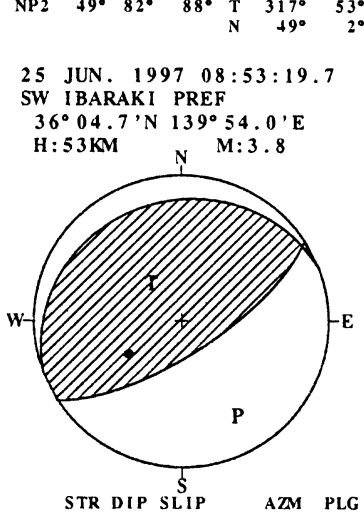

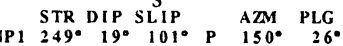

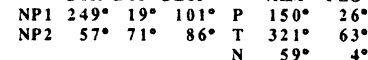
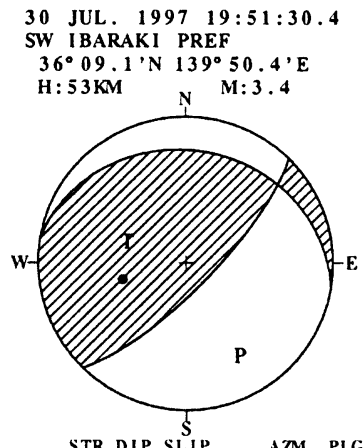

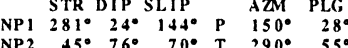

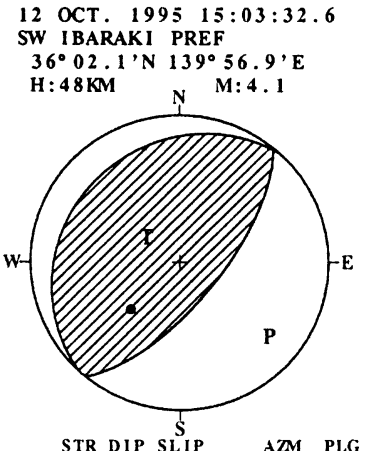

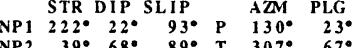

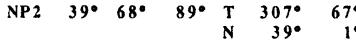

\section{JUN. 1997 18:09:31.3}

$36^{\circ} 04.1$ ' $139^{\circ} 52.0$ '

$\mathrm{H}: 52 \mathrm{KM} N \mathrm{~N} \quad \mathrm{M}: 3.8$

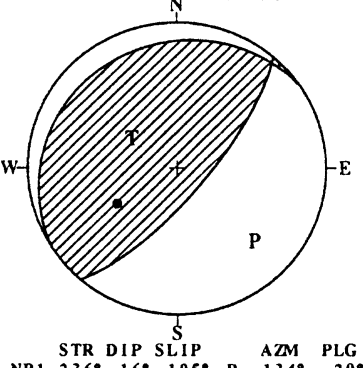

NP1 $236^{\circ} 16^{\circ}$ 105. $105^{\circ}$ A $134^{\circ}$ PLG $\mathrm{NP2} 40^{\circ} \quad 75^{\circ} 86^{\circ} \mathrm{T} \quad 304^{\circ} \quad 60^{\circ}$

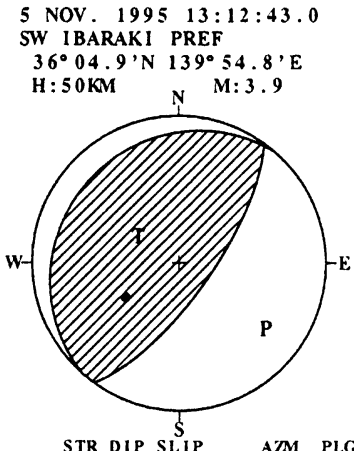

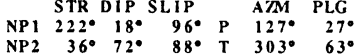

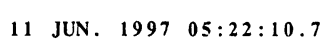
SW IBARAKI PREF

$36^{\circ} 03.4^{\prime} \mathrm{N} 139^{\circ} 53.8$ '

$\mathrm{H}: 51 \mathrm{KM}$

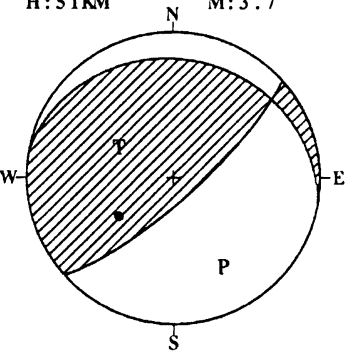

STR DIP SLIP ATM PLG

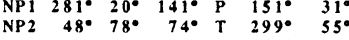

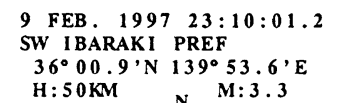

$36^{\circ} 00.9^{\prime} \mathrm{N} 139^{\circ} 53.6^{\prime} \mathrm{E}$

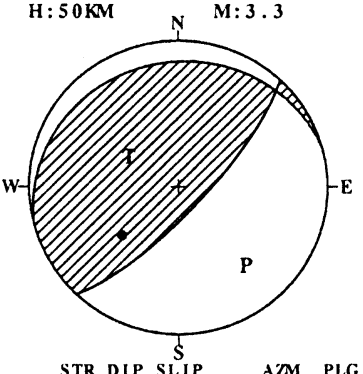

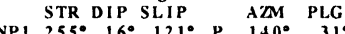

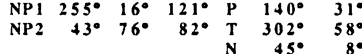

Fig. 9. Focal mechanisms (lower hemisphere) of earthquakes used to investigate the relationship between seismic moment and spectrum ratio. Earthquakes with similar focal mechanisms were selected. A solid circle in the diagram shows the projected position of the Yasato station.

where $G_{0}=\int_{0}^{\infty} g(t) d t$

Moment magnitude $M_{w}$ is calculated from the formula (Kanamori, 1977) as

$$
M_{w}=\left(\log _{10} M_{0}-9.1\right) / 1.5 \text {, }
$$

where $M_{0}$ is seismic moment in Nm. Equation (5) is considered to be applicable to explain the relationship between moment magnitude and a magnitude calculated from regional displacement amplitudes. The coefficient of $M_{J M A}$ to $\log _{10} M_{0}$ is about $2 / 3$ (Utsu, 1982; Katsumata, 1996). It is considered that the relationship between $M_{w}$ and $M_{J M A}$ is an example of the relationship of Eq. (5).

Since Eq. (5) describes the time variation of displacement in far field, the time derivative of $f\left(t, M_{0}\right)$ is considered to be proportional to the velocity.

$$
\frac{d}{d t} f\left(t, M_{0}\right)=M_{0}^{1 / 3} \frac{d g(\tau)}{d \tau} .
$$

The moment dependence in Eqs. (5) and (6) are 2/3 and 1/3, respectively. This means that the ratio $\alpha_{V} / \alpha_{D}$ (Eqs. (1) and (2)) is expected to be 0.5 . The observed value of $\alpha_{V} \simeq 0.8$ is not consistent with this expectation. There may be another scaling law in the source spectrum.

The author tried to make a spectrum model which generates the value of $\alpha_{V} \simeq 0.8$. It is necessary to relate the spectral characteristics to the peak amplitude in the time domain. The method proposed by Boore (1983) is used here to simulate a transient time series. The method for making a transient time series which has specified spectrum density is

1) Make a spectrum model which has random phase and 
unit amplitude for any frequency.

2) Transform the spectrum to the time domain (create a noise series).

3) Apply a shaping time window.

4) Transform the series to the frequency domain.

5) Reject the spectra which have spectral amplitude very different from the unit value at $f=0$.

6) Apply a transfer function with specified spectrum amplitudes.

7) Transform the spectrum to the time domain.

When the transfer function satisfies the causality principle, the time series is practically confined to a finite duration. Since Boore (1983) was interested in the acceleration amplitude, he normalized the time series with a constraint on total energy. In this study, the spectrum density is adjusted at $f=0$, since the amplitude of an earthquake, for which scalar moment is specified, is of interest. Figure 4 shows an example of the synthesized time series.

It is considered that some magnitude-dependent parameter is necessary to be introduced into the spectrum model to derive the value of $\alpha_{V} \simeq 0.8$. The following two-cornerfrequency transfer function is tested for a possible magnitude-dependent spectrum model.

$$
F(\omega)=\frac{1}{1+r} \cdot \frac{1}{\left(1+i \omega / \omega_{1}\right)^{2}}+\frac{r}{1+r} \cdot \frac{1}{\left(1+i \omega / \omega_{2}\right)^{2}},
$$

where $\omega_{1}$ and $\omega_{2}\left(\geq \omega_{1}\right)$ represent angular corner frequencies. This is similar to the two-corner-frequency model proposed by Atkinson and Boore (1995) for earthquakes in eastern North America. Assume here that $\omega_{2} / \omega_{1}$ is magnitudedependent, and $r$ is expressed as a function of $\omega_{2} / \omega_{1}$,

$$
r=\left(\omega_{2} / \omega_{1}\right)^{-s}
$$

If $s$ is 1.0 , the velocity spectrum has 2 peaks of the same height at frequencies of $\omega_{1}$ and $\omega_{2}$. For $s<1.0$, the velocity spectrum has a higher peak at $\omega=\omega_{2}$ than that at $\omega=\omega_{1}$ (Fig. 5). Figure 6 shows the amplitude ratio for $s=0.5$ with $\omega_{2} / \omega_{1}$ on the horizontal axis. The ratio of velocity amplitude increases along with the value of $\omega_{2} / \omega_{1}$, whereas the ratio of displacement amplitude saturates. For $s=1.0$, the slope of the velocity amplitude to $\omega_{2} / \omega_{1}$ is about half of that in Fig. 6.

If it is assumed that the value of $\alpha_{V} \simeq 0.8$ is effective for the earthquakes of magnitude from 3.0 to 6.5 , the amplitude difference ratio of about 11 times could be generated.

$$
\begin{aligned}
\frac{\left(\left.A_{V}\right|_{M_{w}=6.5} /\left.A_{V}\right|_{M_{w}=3.0}\right)_{o b s}}{\left(\left.A_{V}\right|_{M_{w}=6.5} /\left.A_{V}\right|_{M_{w}=3.0}\right)_{s s}} & =\frac{10^{0.8 \times(6.5-3.0)}}{10^{0.5 \times(6.5-3.0)}} \\
& \simeq 11
\end{aligned}
$$

where the suffix of $o b s$ denotes an observed value, and $s s$ an expected value from the self-similar model. It corresponds to $\omega_{2} / \omega_{1} \simeq 13$ in Fig. 6 , and means $\log _{10}\left(\omega_{2} / \omega_{1}\right) \simeq$ $\Delta M_{w} / 3.1 \simeq 0.2 \Delta \log _{10} M_{0}$, where $\Delta$ denotes differences in $M_{w}$ and $\log _{10} M_{0}$. If the value of $\alpha_{V} \simeq 0.8$ covers the magnitude range of 2.5-7.0, the amplitude ratio due to the effect of $\omega_{2} / \omega_{1}$ could reach about 22 .

Some researchers proposed scaling models lack of selfsimilarity, or spectrum models with two corner frequencies (e.g., Aki, 1972; Gusev, 1983; Koyama, 1985; Atkinson and Boore, 1995; Haddon, 1996). Those models are characterized by higher spectrum density in the high-frequency range than that of the $\omega$-square model. Figure 7 shows the source spectrum density ratios between events of $M_{w}=6.6$ and $M_{w}=4.6$ for various models. The spectrum ratios are shown in the figure because the difference in spectrum density between small and big earthquakes is of interest here. The model of this study is consistent with other models in respect of assuming enhanced spectrum density in high-frequency range. But, the spectrum ratio in high frequency of this model is too high comparing with other models, and it might be unrealistic.

The spectrum model indicates that high-frequency signal strength increases with the size of an earthquake. To evaluate this hypothesis, relationship between seismic moment and observed spectrum ratio is examined. The data from earthquakes occurring in the southwest part of Ibaraki Prefecture at depths of about $50 \mathrm{~km}$ is used. Seismicity there is very active due to the interaction between the Pacific plate and the Philippine Sea plate. Figure 8 shows epicenters of analyzed earthquakes. Seismic records obtained at Yasato station (Fig. 8) are analyzed. Earthquakes with similar focal mechanism were selected (Fig. 9). At Yasato station, three types of seismometers were installed, a short-period velocity seismometer, a strong motion accelerometer, and a broad-band seismometer (STS-2). Sampling rates were 100 sample/s for the velocity seismometer and 20 sample/s for the accelerometer and the broad-band seismometer. Cut-off frequencies of anti-alias filters were $40 \mathrm{~Hz}$ for 100 sample/s and $8 \mathrm{~Hz}$ for $20 \mathrm{sample} / \mathrm{s}$.

Figure 10 shows the relationship between moment magnitude and the maximum amplitudes. Moments were estimated by the method of Katsumata (2000). In this case, $\alpha_{V}$ (Eq. (2)) is larger than half the values of $\alpha_{D}$ (Eq. (1)), which means

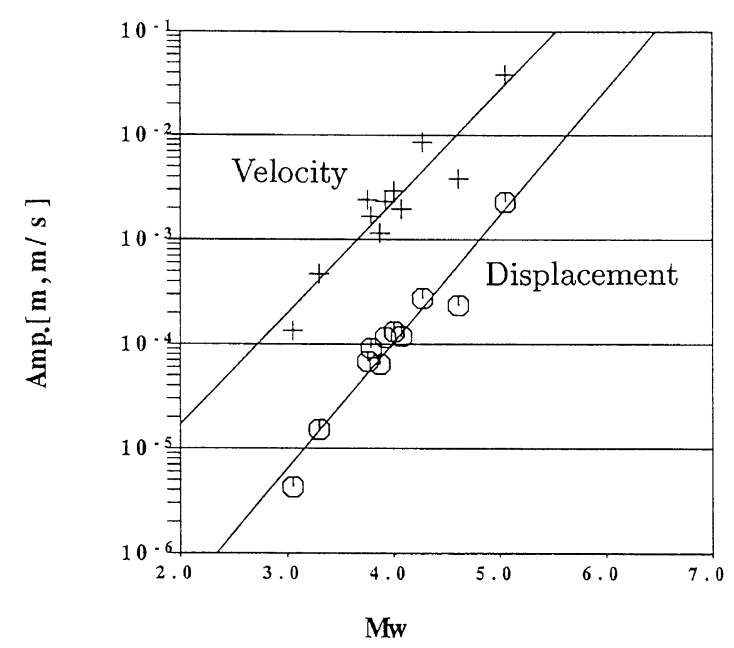

Fig. 10. The maximum displacement (circles) and velocity (crosses) amplitudes of the Yasato records. 

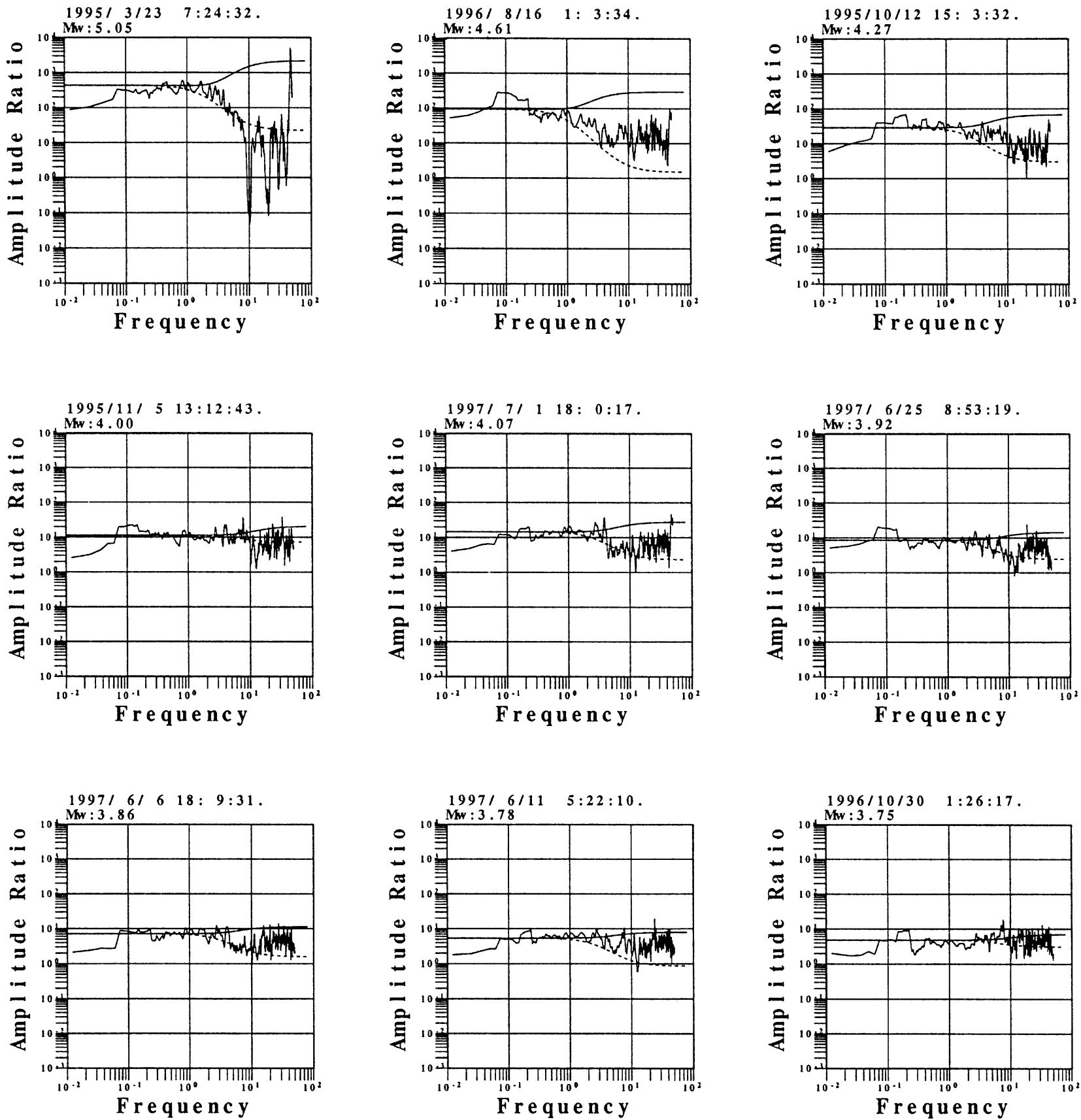

Fig. 11. Spectrum ratios of the records from the events shown in Fig. 9. The record from the earthquake at 23:10 9 Feb. 1997 was used as the denominator The broken curves show the spectrum ratios expected from the $\omega$-square model, and the solid curves the spectrum ratios expected from Eq. (7).

that the relationship between displacement and velocity amplitudes is inconsistent with the self-similar spectrum model.

Spectrum ratios were calculated for these earthquakes using the data from the earthquake of $M_{w}=3.3$ at 23:10 9 Feb. 1997 (JST) as the denominator. This corresponds to deconvolution with an empirical Green's function (Irikura, 1986). This operation virtually excludes path and site effects. Spectrum ratios are shown in Fig. 11. Records obtained from the short-period velocity seismometer were used to calculate the spectrum ratios. Since the records of the short-period and the broad-band instruments were clipped for the earthquake on 23 March 1995, the accelerometer records were used for the earthquake. Records of the accelerometer were interpolated in the calculation of spectrum ratio. The broken curves show the spectrum ratios expected from the $\omega$-square model, and the solid curves indicate the spectrum ratios expected from Eq. (7) with parameters of $s=0.5$ and $\omega_{2} / \omega_{1} \propto M_{0}^{0.2}$. The corner frequency is assumed to be $0.6 / \tau(\mathrm{Hz})$, for which a triangle pulse is assumed. $\tau$ is an observed pulse width of the initial $P$-phase. The $\omega$-square model fits the observations better than the model with enhanced high-frequency components.

\section{Propagation Effects}

In addition to source process, the observed spectrum also expresses the effects of propagation. The propagation trans- 
fer function effects should be independent of earthquake magnitude. Convolution of path effects with the source time function could cause a deviation from the self-similar scaling model.

It is considered that the observed record constitutes the empirical Green's function for examining propagation effects. Synthetic records are calculated here by convolving an observation record as the Green's function with synthetic source time functions. The record of the event of $M_{w}=3.3$ at 23:10 9 Feb. 1997 is used as the empirical Green's function. A synthetic source time function is calculated by the method by Boore (1983), which was used in the previous section. The transfer function $S(\omega)$ for making the synthetic source time function is defined as

$$
S(\omega)=S_{i}(\omega) / S_{0}(\omega)
$$

where $S_{0}(\omega)$ and $S_{i}(\omega)$ are the transfer function for the event of which records are used as the empirical Green's function and that for an event of various magnitudes, respectively. $S_{i}(\omega)$ and $S_{0}(\omega)$ are given as

$$
S_{i}=\frac{1}{\left(1+i \omega / \omega_{c}\right)^{2}},
$$

where $\omega_{c}$ is an angular corner frequency.

Figure 12 shows the relationship between moment magnitude and peak displacement and velocity amplitudes of the synthetic records. The slope of velocity amplitude against magnitude is significantly smaller than that for displacement amplitude, and greater than the half of the slope for displacement amplitude. This shows that propagation effects could cause the apparent deviation from the self-similar scaling model in observed records. It is suggested here that disper-

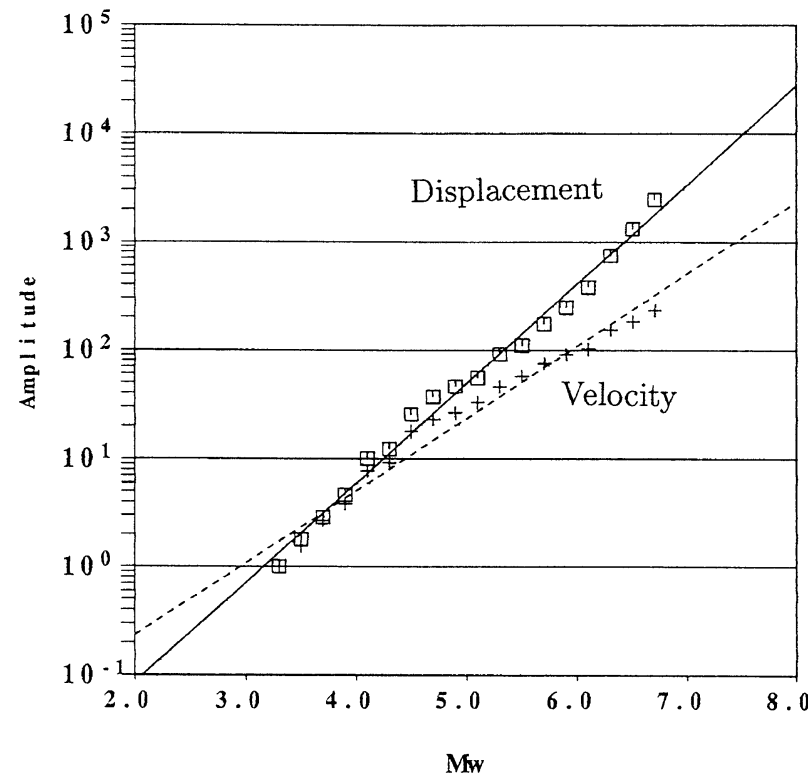

Fig. 12. Relationship between moment magnitude and the peak amplitude of synthetic records obtained with an empirical Green's function and synthetic source time-functions. The square indicates that of displacement amplitude, and the cross indicates velocity amplitude. A solid line in the figure shows the regression line for displacement amplitude, and a broken line that for velocity amplitude. sion and enhancement in high frequencies during propagation cause the deviation from the $\omega$-square model. The plots of velocity amplitudes show a bend at $M_{w} \simeq 4.5$. There is a case in which the bend is of reverse polarity. It is considered that the bend is related to characteristics of spectrum in high frequencies of the sampled earthquake.

The coefficients obtained by Joyner and Boore (1981) $\left(\alpha_{V}=0.489\right)$ and Campbell (1997) $\left(\alpha_{V}=0.51\right)$ are very close to the value expected from the self-similar scaling model. It is considered that those values were obtained for the records so close to the hypocenters that the records were not affected by the propagation very much.

\section{Conclusions}

Relationship between displacement and velocity amplitudes was examined in the time domain. The observed value of the coefficient of the logarithmic velocity amplitude to the logarithmic displacement amplitude was about $0.8-0.9$ for data within $200 \mathrm{~km}$ from hypocenters. The expected value of the coefficient from a self-similar scaling model is 0.5 . It was necessary to assumed very large high-frequency contents to explain the observed value only by a source spectrum model. But the $\omega$-square model fits the observed spectra ratios better than the model of large high-frequency contents. It was shown that the observed value of the coefficient reflected propagation effects by using an empirical Green's function.

Acknowledgments. I wish to acknowledge Prof. Junji Koyama for critical reading of the manuscript. I am grateful to Drs. Kenneth W. Campbell and Saburoh Midorikawa for their constructive reviews.

\section{References}

Aki, K., Scaling law of seismic spectrum, J. Geophys. Res., 72, 1217-1231, 1967.

Aki, K., Scaling law of earthquake source time-function, Geophys. J. R. Astr. Soc., 31, 3-25, 1972.

Aki, K. and P. G. Richards, Quantitative Seismology, 932 pp., W. H. Freeman and Company, New York, U.S.A., 1980.

Atkinson, G. M. and D. M. Boore, Ground-motion relations for eastern North America, Bull. Seism. Soc. Am., 85, 17-30, 1995.

Boore, D. M., Stochastic simulation of high-frequency ground motions based on seismological models of the radiated spectra, Bull. Seism. Soc. Am., 73, 1865-1894, 1983.

Brune, J. N., Tectonic stress and the spectra of seismic shear waves from earthquakes, J. Geophys. Res., 75, 4997-5009, 1970.

Campbell, K. W., Empirical near-source attenuation relationships for horizontal and vertical components of peak ground acceleration, peak ground velocity, and pseudo-absolute acceleration response spectra, Seism. Res. Lett., 68, 154-179, 1997.

Gusev, A. A., Descriptive statistical model of earthquake source radiation and its application to an estimation of short-period strong motion, Geophys. J. R. Astr. Soc., 74, 787-808, 1983.

Gutenberg, B., Amplitudes of surface waves and magnitude of shallow earthquakes, Bull. Seism. Soc. Am., 35, 3-12, 1945a.

Gutenberg, B., Amplitudes of P, PP and magnitudes of shallow earthquakes, Bull. Seism. Soc. Am., 35, 57-69, 1945b.

Gutenberg, B., Magnitude determination for deep-focus earthquakes, Bull. Seism. Soc. Am., 35, 117-130, 1945c.

Haddon, R., Earthquake source spectra in eastern North America, Bull. Seism. Soc. Am., 86, 1300-1313, 1996.

Irikura, K., Prediction of strong acceleration motion using empirical Green's function, Proc. 7th Japan Earthquake Eng., 8, 37-42, 1986.

Japan Meteorological Agency (Earthquake Disaster Prevention Section, Earthquake and Tsunami Observation Division, Seismological and Volcanological Department), Data processing of electromagnetic strong motion seismographs, Technical Correspondence of Seismological and Volcanological Department, No. 65, 35-58, 1989 (in Japanese).

Joyner, W. B. and D. M. Boore, Peak horizontal acceleration and velocity 
from strong motion records including records from the 1979 Imperial Valley, California, Earthquake, Bull. Seism. Soc. Am., 71, 2011-2038, 1981

Kakishita, T., I. Sasakawa, M. Kobayashi, A. Nagai, and N. Hamada, An examination of JMA formulas for determining magnitude using data obtained by the JMA- 87 type electromagnetic strong motion seismograph, Zisin, II, 45, 263-277, 1992 (in Japanese with English abstract).

Kanamori, H., The Energy Release in great earthquakes, J. Geophys. Res., 82, 2981-2987, 1977.

Kanbayashi, Y., Some problems underlying the empirical formulas for determining the magnitude of the shallow focus earthquake using the JMA 67 and 76 type seismographs, Quart. J. Seism., 55, 81-88, 1992 (in Japanese with English abstract).

Katsumata, A., Automatic designing of Bessel digital filters, Quart. J. Seism., 56, 17-34, 1993 (in Japanese).

Katsumata, A., Comparison of magnitudes estimated by the Japan Meteorological Agency with moment magnitudes for intermediate and deep earthquakes, Bull. Seism. Soc. Am., 86, 832-842, 1996.

Katsumata, A., Seismic moment estimation of small earthquakes by waveform fitting, Pap. Meteorol. Geophys., 51, 1-15, 2000.

Koyama, J., Earthquake source time-function from coherent and incoherent rupture, Tectonophys., 118, 227-242, 1985.

Midorikawa, S., Preliminary analysis for attenuation of peak ground velocity on stiff site, Proceedings of the International Workshop on Strong Motion Data Vol. 2, 39-48, 1993.

Molas, G. L. and F. Yamazaki, Attenuation of earthquake ground motion in Japan including deep focus events, Bull. Seism. Soc. Am., 85, 1343-1358, 1995.

Richter, C. F., An instrumental magnitude scale, Bull. Seism. Soc. Am., 25 $1-32,1935$

Tsuboi, C., Determination of the Gutenberg-Richter's magnitude of earthquakes occurring in and near Japan, Zisin, II, 7, 185-193, 1954 (in Japanese with English abstract).

Utsu, T., Relationships between earthquake magnitude scales, Bull. Earthq Res. Inst., Univ. Tokyo, 57, 465-497, 1982 (in Japanese with English abstract).

Watanabe, H., Determination of earthquake magnitude at regional distance in and near Japan, Zisin, II, 24, 189-200, 1971 (in Japanese with English abstract).

Yoshioka, K. and Y. Iio, Re-examination of magnitude's formula for records of velocity seismograph with natural frequency of $1 \mathrm{~Hz}, \mathrm{Zisin}, \mathrm{II}, \mathbf{4 1}$ 437-441, 1988 (in Japanese).

A. Katsumata (e-mail: akatsuma@typhoon.mc-jma.ac.jp) 